\title{
Tradición literaria y tratamiento del espacio en la narrativa boliviana contemporánea. Una lectura a partir de Lluvia de piedra de Rodrigo Urquiola Flores
}

(1) Magdalena González Almada

CIFFyH-CONICET / Universidad Nacional de Córdoba, Argentina

\begin{abstract}
Resumen
El presente artículo se propone como un análisis de Lluvia de piedra (2011), novela del autor boliviano Rodrigo Urquiola Flores (1986) en la cual aparece como espacio privilegiado la ciudad de La Paz en tensión con Antofagasta.

El análisis se realiza a partir de dos vectores: en primer lugar, la relación que este texto sostiene con la tradición literaria boliviana y, en segundo lugar, la relevancia que el tratamiento del espacio asume en el mismo. La revisión de la tradición literaria boliviana permite observar en qué medida la narrativa de Urquiola Flores se tensiona con ella, las recurrencias a las cuales apela y la actualización de los imaginarios sociales que atraviesan la literatura boliviana. En este mismo sentido, se examina la impronta del espacio como una noción que se presenta productiva al momento de analizar la narrativa boliviana contemporánea de tema paceño. Asimismo, este análisis permite revisar el concepto de nación a partir de la configuración que del espacio realiza Urquiola en su novela.
\end{abstract}

Este trabajo apela a marcos teóricos vinculados con la noción de territorio (Calomarde, Ighina) y a trabajos de la crítica centrados en el análisis de la tradición literaria boliviana (Antezana, Wiethüchter, González Almada).

PALABRAS CLAVE: Novela boliviana contemporánea, Rodrigo Urquiola Flores, tradición literaria, espacio

Literary tradition and treatment of space in contemporary Bolivian narrative. A reading from the Lluvia de piedra by Rodrigo Urquiola Flores

\footnotetext{
Abstract

This article is proposed as an analysis of Lluvia de piedra (2011) written by the Bolivian author Rodrigo Urquiola Flores (1986) novel in which the city of La Paz appears as a privileged space in tension with Antofagasta.
} 
The analysis will be made from two vectors to highlight: first, the relationship that this text supports with the Bolivian literary tradition and, secondly, the relevance that the treatment of space assumes in it. The revision of the Bolivian literary tradition will allow us to observe the extent to which Urquiola Flores's narrative is stressed with her, the recurrences to which she appeals and the updating of the social imaginary that crosses Bolivian literature. In this same sense, the imprint of space will be analyzed as a notion that is presented productive when analyzing the contemporary Bolivian narrative of La Paz. Likewise, this analysis will allow revising the concept of nation based on the configuration that Urquiola makes of space in his novel.

This work will appeal to theoretical frameworks linked to the notion of territory ( Calomarde, Ighina) and criticism works that focus on the analysis of the Bolivian literary tradition (Antezana, Wiethüchter, González Almada).

KEYWORDS: Contemporary Bolivian novel, Rodrigo Urquiola Flores, literary tradition, space

\section{Tradição literária e tratamento do espaço na narrativa boliviana contemporânea. Uma leitura da Lluvia de piedra de Rodrigo Urquiola Flores}

\section{Resumo}

O presente artigo propõe-se como uma análise de Lluvia de piedra (2011) escrita pelo autor boliviano Rodrigo Urquiola Flores (1986) romance no qual aparece como espaço privilegiado a cidade de La Paz em tensão com Antofagasta.

A análise será realizada partindo de dois vetores a destacar: em primeiro lugar, a relação que este texto sustenta com a tradição literária boliviana e, em segundo lugar, a relevância que o tratamento do espaço assume no mesmo. A revisão da tradição literária boliviana permitirá observar em que medida a narrativa de Urquiola Flores se tenciona com ela, as recorrências às quais apela e a atualização dos imaginários sociais que atravessam à literatura boliviana. Nesse sentido, se analisarão as marcas do espaço como noção que se apresenta produtiva ao momento de analisar a narrativa boliviana contemporânea de tema pacenho. Assim mesmo, esta análise permitirá conferir o conceito de nação a partir da configuração que do espaço realiza Urquiola nos seu romance.

Este trabalho apelará a marcos teóricos vinculados com a noção de território (Calomarde, Ighina) e aos trabalhos de crítica que se centram na análise da tradição literária boliviana (Antezana, Wiethüchter, González Almada).

PALAVRAS CHAVE: Romance boliviano contemporânea, Rodrigo Urquiola Flores, tradição literaria, espaço 


\author{
Mi única esperanza la tuve a solas conmigo en la playa. \\ No sé si era un recuerdo o algo más alejado. \\ Entonces, \\ en mis ojos cerrados su cuerpo de miel naufragaba. \\ Oscar Cerruto, "Poema del mar imaginario"
}

\title{
Introducción
}

La narrativa boliviana contemporánea publicada desde los primeros años del siglo XXI ha tomado posiciones diversas en lo que respecta a la tradición literaria del siglo pasado. La crítica abordó estos temas desde varios puntos de vista en los cuales es posible evidenciar algunas recurrencias en la conformación de un campo literario y en la constitución de una tradición que pervive hasta nuestros días. En este sentido, es importante definir cuáles fueron los lineamientos que condujeron a la confección de dichos estatutos, las preocupaciones que las motivaron, los intereses puestos en juego. Es interesante observar, al respecto, que la preocupación por la "nación boliviana", la cristalización de los imaginarios que la conformarían y las delimitaciones territoriales que la integrarían son temas que no han quedado por fuera de la composición literaria, principalmente durante la primera mitad del siglo XX.

La crítica literaria, en efecto, visitó estos temas con frecuencia y en diversos momentos. La pregunta que circula en artículos escritos por Blanca Wiethüchter (1986) y por Luis Antezana (1993) ronda el cuestionamiento sobre las motivaciones que subyacían a las novelas consideradas como canónicas de los años 1930 y 1940. La preocupación por una definición de nación, de sujeto nacional y de territorio nacional, incluso, avivan las discusiones sobre la relación que la producción literaria contemporánea sostiene con el pasado de la literatura del país andino-amazónico.

Si bien muchos de los imaginarios sociales que la literatura publicada a lo largo del siglo XX contribuyó a sostener tuvieron como centro de su preocupación la "problemática nacional", la literatura publicada a lo largo del siglo XXI cuestionó, se alejó y complejizó la pregunta por la conformación de la tradición literaria nacional.

El tratamiento del espacio en la narrativa boliviana, tanto durante el siglo XX como el XXI, resulta una llave que habilita un análisis complejo de la producción literaria contemporánea. El territorio nacional y la actualización de algunos imaginarios relativos a él son tratados por algunos textos de manera compleja, reuniendo en sus tematizaciones retazos de la historia (González Almada, 2017) e impugnaciones que colocan los hechos narrados en territorio extranjero (González Almada, 2016).

Este artículo abordará la novela de Rodrigo Urquiola Flores Lluvia de piedra publicada en el año 2011 y la vinculará con la tradición literaria boliviana al mismo tiempo que expondrá su particular tratamiento del espacio en relación con la narrativa de los siglos XX y XXI.

\section{Tradición literaria y tratamiento del espacio}

Nancy Calomarde (2017) realiza una cartografía del tratamiento que recibió la categoría territorio en la crítica literaria latinoamericana de la segunda mitad del siglo XX. En dicho mapeo, la autora presenta conceptos vinculados con la noción de territorio tales como región y nación, lo que da cuenta de una estrecha relación entre estas categorías. El énfasis de Calomarde está puesto en la relación casi indiscernible entre territorio -espacio-, conformación de la subjetividad y escritura. Domingo Ighina (2005), por su parte, afirma que 
los estados-nación en América Latina se fundaron antes que en una situación de hecho, devenida de un reclamo de continuidad cultural y social o de una circunstancia política y militar definitiva, en proyectos, o red de proyectos, intelectuales, cuyo objeto lo constituía el espacio (2005: 14)

lo que equivale a sostener que la relación que indica Calomarde (2017) es clave para la comprensión de la apropiación de los espacios en América Latina. Ighina, asimismo, introduce la categoría "diseños territoriales", la cual supone la evidencia de

\begin{abstract}
planificaciones de un territorio no siempre correspondiente con el espacio que efectivamente ocupaba el estado, pero que indefectiblemente indicaría los límites deseados por la futura nación, tanto en lo económico, en lo político como en lo objetivado en los símbolos nacionales. [...] El territorio forma un mapa convencional de porciones geográficas aceptado por un estado (en este sentido es el llamado espacio nacional), al tiempo que constituye un diseño intelectual de apropiación de ciertos significados espaciales como solar, nación, continente. (2005: 14-15)
\end{abstract}

El trabajo de investigación de Ighina proporciona una herramienta muy productiva para el presente estudio ya que allana el camino para la comprensión del particular modo de apropiación y organización del espacio en el marco del estado-nación republicano en Bolivia. Durante el periodo republicano iniciado en 1825 con la emancipación de la corona española, el Estado boliviano sostuvo sus límites territoriales en un espacio que, por un lado, imprimió en sus tierras el diseño territorial de las élites, las cuales desconocieron toda organización del espacio anterior a la república. Esto equivale a decir que la organización territorial de los diversos pueblos indígenas que lo ocupaban quedaba sin efecto. De otra parte, ese mismo territorio sufrió graves embestidas entre fines del siglo XIX y la primera mitad del siglo XX. La Guerra del Pacífico (1879-1883) que enfrentó a bolivianos y peruanos contra chilenos, la Guerra del Acre (1899-1903) contra brasileños y la Guerra del Chaco (1932-1935) contra paraguayos, supusieron -en conjunto- sendas pérdidas de territorio en casi todos los límites fronterizos de Bolivia durante ese periodo. La nueva configuración del espacio nacional, más allá de las pérdidas económicas y comerciales, conllevaba a una flagrante pérdida territorial que tuvo como consecuencia una profunda proyección en los imaginarios sociales y en la instalación de matrices discursivas referidas al vínculo entre nación y territorio. Así, el propio Alcides Arguedas en el ensayo positivista Pueblo enfermo [1909] discursiviza la frustración experimentada por las clases mestizas letradas y por las oligarquías bolivianas frente al innegable número de habitantes indígenas y la imposibilidad de reproducir modelos argentinos o chilenos de exterminación del componente originario y recepción de migrantes europeos:

De no haber predominio de sangre indígena, desde el comienzo habría dado el país orientación consciente a su vida, adoptando toda clase de perfecciones en el orden material y moral, y estaría hoy en el mismo nivel que muchos pueblos más favorecidos por corrientes inmigratorias venidas del viejo continente. (s/f: 32 )

Más tarde, y en el mismo ejercicio ensayístico, Arguedas expresa:

Alejada la nación del mar y cerrada dentro del Continente por la muralla de los Andes, no hubo desde entonces la posibilidad de que el elemento étnico se renovase merced al contacto con gentes de otras razas y cambiase de esa suerte la estructura de su misma composición, como fatal y necesariamente ha sucedido con los pueblos de la costa, muchos de los cuales ofrecen hoy una homogeneidad envidiable. (62)

El imaginario del enclaustramiento, caro a numerosos pensadores y ensayistas bolivianos, permeó de manera profunda en la constitución de la subjetividad boliviana como 
un atributo negativo, de atraso y de falta de progreso. Sin la necesaria incorporación del elemento extranjero que procediera de los puertos, el predominante componente indígena se convertía en la causa del atraso y del oprobio frente a las naciones del mundo. Es decir, y retomando lo expresado hasta el momento, la configuración territorial nacional de Bolivia hacia los años 40 del siglo pasado estuvo fuertemente atravesada por las pérdidas territoriales y por la subordinación de la milicia boliviana a otras extranjeras. El recuerdo de estas pérdidas militares que impactaron en la definición de los límites de la nación andino-amazónica tallaría la identidad boliviana.

Otro aspecto insoslayable en lo que refiere a la discusión por el tema del territorio en Bolivia tiene relación con la cuestión de las tierras altas y de las tierras bajas. En efecto, se trata de un país que se ha configurado a partir de la generación de un imaginario habitado por el predominio de las tierras altas, del altiplano boliviano, del occidente del país. Más relegada y con escasa participación política, la región de las tierras bajas, el oriente boliviano, ha intentado irrumpir en la vida política boliviana, a partir de la segunda mitad del siglo XX, con fuertes proyecciones hacia el presente siglo. ${ }^{1}$

En el marco del esquema territorial y político descrito, el campo literario boliviano se hizo eco de estas discusiones y tomó la ficción literaria como palestra para exponer puntos de vista y lineamientos que interpelaban la realidad social y política. Aunque suele considerarse por parte de la crítica especializada que este tipo de objetivo estético jugaba las veces de sustituto frente a la vacancia en la producción intelectual de las ciencias sociales (Antezana, 2000), es, no obstante, el discurso literario el privilegiado para el debate de ideas. Muchas de ellas estaban inclinadas a metaforizar diversos proyectos de nación, los sujetos nacionales que debían integrarla, la discusión por el indígena, por el lugar de las diversas clases sociales, etc. Una considerable cantidad de textos fueron escritos al calor de los acontecimientos históricos y, en efecto, fue la Guerra del Chaco la que inspiró, con mayor frecuencia, numerosos cuentos y novelas que abordaban el tema bélico y sus implicancias en la vida social y cultural boliviana.

La crítica literaria y poeta Blanca Wiethüchter (1986) observa que

La literatura boliviana debe ser situada, primero, como proyecto de fundación de una elite criolla ligado a la formación de una nación que se quiere autónoma (no tenemos sino ciento sesenta años de independencia), y segundo, que este proyecto no puede sino entenderse como un polo de la dicotomía criollo-indígena. Que esta polarización está sujeta a una notoria contradicción, que, además de manifestarse en la literatura criolla, se expresa en la literatura oral indígena. (1986: 2)

Literatura indígena que, por cierto, no ha sido demasiado visibilizada ni legitimada por la academia boliviana. ${ }^{2}$ En todo caso, la crítica especializada marca con recurrencia que es desde la incursión literaria de Marcelo Quiroga Santa Cruz con la novela Los deshabitados y de Oscar Cerruto con la publicación del volumen de cuentos Cerco de penumbras, ambos publicados en el año 1957, cuando se abandona el modelo realista y costumbrista que predominaba en el campo literario de la primera mitad del siglo XX.

1 Para conocer más sobre la historia del desarrollo del oriente boliviano y sus proyecciones sociales, ver Prudén, $\mathrm{H}$. (2018): “Apuntes para una historia del mestizaje en Santa Cruz de la Sierra, Bolivia”, Revista Iberoamericana, año XVIII, n. 67, Berlín, disponible en https://journals.iai.spk-berlin.de/index.php/iberoamericana/article/view/2446/2020

2 La producción literaria publicada por autores indígenas se ha visibilizado recientemente y ha provocado diversos trabajos de la crítica. Entre otros es posible consultar González Almada, M. (2017) “Abigarramiento lingüístico, resistencia y traducción: la poesía de Mauro Alwa en el contexto de la literatura boliviana contemporánea” en Mitologías hoy. Revista de pensamiento, crítica y estudios literarios latinoamericanos, vol. 16, Barcelona, disponible en https://revistes. uab.cat/mitologias/article/view/v16-gonzalez/48o-pdf-es, y Ayllón, V. (2007) “Aruskipasipxañanakasakipunirakispawa: notas sobre dos poetas indígenas bolivianos” en Revista Nuestra América, n.ํㅜ, Porto disponible en https://core.ac.uk/ download/pdf/61009959.pdf 
Es cierto que este campo estaba compuesto por textos y autores que hegemonizaban el sistema literario con sus temas pese a que otros autores y autoras disputaban la centralidad de ese campo, aunque no con buenos resultados. Sin embargo, es preciso analizar el campo literario para poder discernir las tensiones e impugnaciones que lo atraviesan. Raymond Williams provee una clave para reflexionar sobre este asunto. De acuerdo al desarrollo que realiza en Marxismo y literatura (2009), el autor indica que la tradición es el pasado sobreviviente que, de todos modos, "es visto, de hecho, como una fuerza activamente configurativa, puesto que la tradición es en la práctica la expresión más evidente de las presiones y límites dominantes y hegemónicos" (2009:158-159), lo cual redunda en la configuración de una tradición selectiva en la que permanecen ciertos textos y autores y otros son condenados al olvido.

En consonancia con estas cuestiones, Gina Saraceni (2008) propone que la relación con el pasado, en este caso literario, supone un conflicto que es necesario examinar y-en muchos casos- recomponer a partir de la memoria y de la asunción de una herencia que no es recibida sin más. Por el contrario, se trata de una revisión permanente que agita las aguas calmadas del campo literario hegemonizado para imprimirle un oleaje que embate los pilares de la tradición literaria en Bolivia.

En el contexto de la narrativa boliviana contemporánea se evidencia un momento literario que inaugura, a comienzos del siglo XXI, una variedad de aspectos y de temas que renuevan el campo. Muchos de los autores y autoras han abandonado las motivaciones que tuvieron sus "padres literarios" y, en ese sentido, vehiculizan otras preocupaciones, muchas de ellas vinculadas con la cuestión del territorio. En ese sentido, el tratamiento del espacio adquiere mayor énfasis y solidez al presentar una serie de recurrencias que constituyen, en sí mismas, nudos de análisis literarios que en otras oportunidades ya han sido revisadas (González Almada, 2017 y 2016). En todo caso, y en relación con la preocupación que convoca a este trabajo, es preciso observar el modo en el cual se actualiza la tradición y algunos de sus temas. Para ello, la lectura de la novela de Rodrigo Urquiola Flores Lluvia de piedra (2011) será referencial.

\section{Lluvia de piedra y la actualización de los imaginarios vinculados con el espacio y la tradición literaria}

Rodrigo Urquiola Flores narra la anécdota de su novela de este modo:

Un viejo, Esteban, retorna a La Paz para encontrar el perdón de su víctima, Marianela, antigua novia suya, a quien había asesinado a patadas.

En la estación de trenes - siempre quise que La Paz conservara su estación de trenes-, Esteban se encontró con una Marianela que había quedado congelada en el tiempo, o sea, que no había envejecido como él; se encontró con una mujer a la que la muerte había salvado morir. (2016: 116)

La novela de Urquiola se estructura en dos partes que marcan el derrotero de Esteban. La primera parte, denominada "El ascenso", contiene ocho capítulos numerados de manera ascendente, mientras que la segunda parte, "El descenso", contiene cinco capítulos numerados de manera descendente.

La importancia de la memoria como agente catalizador de las acciones narradas resulta productiva para comenzar el análisis del texto, en tanto que este eje atraviesa toda la novela y, de algún modo, la determina. Esteban vuelve de Antofagasta a operar una "reconstrucción", como es mencionado en reiteradas ocasiones en el texto: "reconstruirlo todo. A ti, Marianela, y a ti, mi casa" (2011:74). La idea de reconstrucción está 
ligada a la de memoria. Mientras que la memoria aparece en el texto como un ejercicio de redención y alivio para el personaje principal, la idea de reconstrucción funciona como la parte material de esa memoria, imprimiéndose en los objetos. Se quiere reconstruir la casa, la antigua casa en la que vivían los enamorados como fórmula para volver en el tiempo y, también, con la intención de restaurar un orden perdido. Es ese orden perdido el que parece escurrírsele al protagonista, es una reconstrucción que nunca llegará a darse.

Víctima del remordimiento y de la culpa, Esteban huye a Antofagasta luego de dar muerte a su novia. Este lugar que ahora forma parte de Chile, remite a la Guerra del Pacífico y a la pérdida de territorio como consecuencia de la misma. Fundada por el gobierno boliviano en 1868 , ya desde tiempos más antiguos funcionaba como puerto de desembarque y de descanso para los chilenos. La historia de Antofagasta indica que pasa de puerto boliviano a posesión chilena y, con este hecho, también se transfieren todas las riquezas minerales y salitreras en favor del país transandino. Como ya fue mencionado con anterioridad, la Guerra del Pacífico pervive en el imaginario boliviano hasta la actualidad y este recuerdo de pérdida atraviesa la subjetividad de los bolivianos. En ese sentido, es llamativo que, para Esteban, Antofagasta sea el lugar del escape, aunque no consiga nunca poder apropiarse, territorializarse, ${ }^{3}$ en esa ciudad: "por fin había logrado comprar una casa en una ciudad a orillas del mar, en Antofagasta. [...] él, dentro de todo lo que había conseguido, no podía dejar de sentirse extranjero" (2011: 18-19). Como el propio texto lo explicita, Esteban se siente "demasiado extranjero, doblemente forastero" (19) en tanto que el personaje no se encuentra en Bolivia, aunque Antofagasta haya sido en el pasado un lugar boliviano. Antofagasta le recuerda el trauma de la pérdida del territorio y, además, se construye en la novela una extranjería que acentúa la imposibilidad del personaje de despegarse de su pasado. Así, el pasado parece perseguirlo sin descanso, pese a que los intentos por borrarlo y generar una nueva vida fuera de Bolivia -aunque Antofagasta pudiera significar Bolivia en algún sentido- no pueden traerle paz al personaje.

Las menciones al mar y a los objetos que se relacionan con él: "el aroma salino del agua marina" (21), "era probable que al mar le gustara viajar en tren" (21), se relacionan con el viaje: "el viaje lo era todo" (18) y reafirman el recuerdo del territorio perdido. Sin embargo, la extranjería deja de representarse como tensión una vez que Esteban vuelve a Bolivia. Cuando el personaje retorna a la ciudad de La Paz se inicia el proceso de "reterritorialización" (Guattari y Rolnik, 2013), es decir, comienza un momento de reapropiación del territorio paceño, de enraizamiento con el espacio que considera su casa (Urquiola Flores, 2011: 24). En consecuencia, el personaje de Lluvia de piedra transita entre dos territorios bolivianos: uno, Antofagasta, perdido, irrecuperable; el otro, La Paz, se erige como el territorio de lo posible. Esta línea de reflexión conduce a pensar que la redención y reconstrucción a la que aspira el personaje solo es posible en Bolivia, en la actual Bolivia, no en el territorio perdido. La tensión planteada entre lo recuperable y lo reconstruíble subyace toda la novela, otorgándole al personaje de Esteban un soporte sobre el cual apoyarse.

La breve mención a Antofagasta se intercambia con La Paz en un trueque que muda pasado por presente. Si Antofagasta es el espacio del presente muy pronto, mediante el viaje, se volverá parte del pasado, mientras que La Paz y los sucesos acaecidos en ese tiempo trocarán pasado por presente al volverse el presente de la narración. Aunque en algún momento Esteban guardará la esperanza de volver a la ciudad costera, este plan nunca se concreta. Más bien, Esteban encontrará su redención -su propia muerte- en La Paz y en la casa que su ilusión quiso reconstruir. 
Desde la perspectiva de este análisis, el personaje compuesto por Rodrigo Urquiola se presenta como una apuesta literaria que pretende tensionar lo que refiere a la "realidad" y a la imaginación. El tenue límite que se tensiona en Esteban lo conduce, en ocasiones, a ser un personaje que confunde algunas dimensiones, es decir, se trata de un personaje que tanto podría haber imaginado los sucesos narrados como podría haberlos experimentado. La sutileza narrativa de Urquiola actualiza uno de los problemas más discutidos de la literatura: la representación de lo real y su relación con la ficción. En el caso de Lluvia de piedra se trata de poner en tensión más bien los "efectos de realidad" que irrumpen en el conflicto narrado. Es posible admitir que se trata de una imaginación, ${ }^{4}$ la del personaje, que le posibilita materializar y construir una realidad en acuerdo con aquello que ha imaginado. Esto sugiere que Esteban habita un espacio intersticial en el que la "realidad" es un constructo que lo contiene y que le permite transitar tanto lo imaginado como el mundo construido por fuera de esa imaginación. En ese sentido, Urquiola plantea un interesante juego entre "realidad" y ficción e, incluso, presenta una propuesta narrativa que se instala en lo "metaficcional" dado que es posible considerar que, dentro del marco de la ficción literaria, el personaje crea una ficción para sí mismo. Como en un juego de encastre, una "realidad" se coloca dentro de otra y, en Esteban, esto adquiere diversas intensidades a lo largo del texto. Ello resulta en una de las características más relevantes del personaje a la vez que es el catalizador del conflicto de la novela. Esteban, entonces, es un personaje que imagina y aquello que imagina, en ocasiones, se vuelve, como se verá más adelante en la novela, el disparador del delirio y, también, del desquicio. La confusión entre lo "imaginado" y lo "real" hace que Esteban se ubique en el delirio de su propia ambición desbordada: la reconstrucción de la casa, de la relación con Marianela, de su propia subjetividad. En el relato se imprime un dejo de enloquecimiento en el personaje motivado por la imaginación activa:

Recordó que alguna vez había imaginado el encuentro de la muerte con el aliento vital de su abuela y ahora quiso ver cómo habría ocurrido este mismo encuentro con el aliento vital de Marianela. [...] Prefirió imaginar que ese instante de verlo todo negro y luego ya no ver nada, ni sentir nada, ni ser nada era el umbral por el que ella ingresaba a otro mundo hecho -como este primero- de imaginación. (2011: 138-139)

Esta rara circunstancia de indefinición o, mejor, de vacilación entre lo "real" y lo "imaginado", de clara influencia tomada del género fantástico, sostiene la trama de la novela. Durante la primera parte, la presencia de Marianela parece real, es decir, la difunta se presenta como si nunca lo hubiera estado y acompaña a Esteban en la casa, le habla, le cocina. La desaparición de Marianela en la segunda parte de Lluvia de piedra somete a Esteban en la desesperación; la duplicidad y la tensión entre realidad e imaginación se profundiza y se consolida la decadencia del personaje.

La vacilación propiciada por la atmósfera creada en el contexto del género fantástico conecta la novela de Urquiola con la tradición literaria boliviana. El nexo más evidente se establece con el cuento "El círculo" de Oscar Cerruto, publicado-como ya fue mencionado- en 1957 en el volumen Cerco de penumbras.

La anécdota del cuento de Cerruto podría resumirse en lo siguiente: Vicente vuelve de un viaje de dos años a encontrarse con su novia con quien tenía una tensa relación de disputas. Elvira, con su carácter temperamental, de todos modos enamoró a Vicente quien decide partir sin despedirse. Al retornar, el muchacho visita a su enamorada y el encuentro se realiza en términos amorosos y sin peleas. Aunque el ambiente está muy frío y hay señales de que algo extraño está pasando, sobre todo por el carácter

4 Recupero aquí imaginación como "la facultad de representarse las cosas en el pensamiento y con independencia de la realidad" (Cabrera, 2008: 17). 
apaciguado de la novia, Vicente deja un reloj de regalo a Elvira y promete volver al día siguiente. Cuando lo hace, los vecinos le anuncian que la mujer ha muerto hace meses y que resulta imposible que el encuentro de la noche anterior hubiera tenido lugar.

"El círculo" introduce ya en la narrativa boliviana el tema de la vida y de la muerte en una lógica que, de todos modos, no es muy ajena a los imaginarios vinculados con la muerte en la literatura del país andino-amazónico. De hecho, en la narrativa boliviana contemporánea, continúa siendo uno de los temas privilegiados y es posible encontrarlo en diversos textos de Liliana Colanzi, Juan Pablo Piñeiro e incluso en Giovanna Rivero con la introducción del zombie en la narrativa boliviana. De esta manera, la filiación con la tradición literaria de Lluvia de piedra es doble: en cuanto al género, porque se emparenta con el fantástico cerrutiano mediante la anécdota y la delgada línea trazada entre vida y muerte; en cuanto a la recurrencia, porque forma parte de una extensa constelación de escritores en el tratamiento del tema de la muerte que lo incluye en la estela de Jaime Sáenz (1921-1986) y de Jesús Urzagasti (1941-2013).

En este sentido, entonces, Lluvia de piedra presenta un tratamiento del espacio que actualiza el recuerdo del territorio perdido, como perdido se encuentra el personaje, desterritorializado frente a circunstancias de difícil explicación, la confusión de la muerte y de la violencia que no se dieron lugar en Antofagasta sino en La Paz. En el juego de dobles que propone Urquiola, el espacio de la violencia y de la muerte corresponde al territorio que le fue arrebatado a Bolivia, pero el autor invierte esa relación y decide colocar estos elementos en la ciudad donde Esteban se siente como en su casa (2011: 24) tal como aparece enunciado en la última oración del primer capítulo y en la primera del segundo capítulo de la primera parte. "Estoy en casa" (26-27) es para Esteban no solo la posibilidad de un descanso sino, y sobre todo, la ocasión de reconstruir y reversionar los hechos violentos que tuvieron como víctima a Marianela.

\section{Reflexiones finales}

Lluvia de piedra (2011) de Rodrigo Urquiola Flores es una novela que actualiza algunos imaginarios vinculados con el espacio en la narrativa boliviana contemporánea. La reminiscencia del territorio perdido durante la Guerra del Pacífico, la insistencia en la evocación del mar y la expresión de su nostalgia, así como también la configuración de la ciudad de La Paz como el territorio propicio para el establecimiento de un ambiente impregnado por lo fantástico, hacen de este texto un eslabón integrante de la cadena de una literatura que se escribe desde el pasado y lo actualiza.

La insoslayable relación que el texto de Urquiola Flores establece con la tradición literaria, la boliviana pero también la latinoamericana, emparentan a Lluvia de piedra con una literatura que se inscribe en ella sin aislarse de la tradición y acudiendo a ella con nuevas motivaciones. La renovación del género fantástico recreado por el autor paceño toma elementos de la materia histórica así como también apela a un imaginario social ampliamente extendido en Bolivia vinculado con la pérdida soberana de la salida al mar. Ese sentimiento de pérdida se enlaza con las circunstancias que atraviesa Esteban, lo cual le aporta profundidad al personaje.

Urquiola propone con su novela la posibilidad de realizar un pacto con la imaginación. Si Esteban navega indistintamente entre la realidad y las representaciones ilusorias que se manifiestan en el plano de lo imaginario, es porque el personaje busca de manera infructuosa reconstruir aquello que fue destruido. El límite difuso entre las dialécticas de lo real/imaginario y del proceso de construcción/destrucción/ reconstrucción aspira a exponer la vacilación del personaje que transita los espacios del pasado víctima del remordimiento y del arrepentimiento. Estos espacios, 
reconstruidos desde la memoria con el propósito de redimir al personaje, vuelven a territorializarlo, a ubicarlo en el lugar en el que se fueron desencadenando los sucesos que llevaron a la muerte de Marianela. Así Esteban acaba afirmándose en la delgada línea que su imaginación ha construido con el fin de trascender la culpa, la de la muerte de Marianela y la del abandono.

En Lluvia de piedra, finalmente, se inscriben los arrebatos que encuentran en el delirio y la locura su máxima expresión. Esteban, como Vicente, el viajero que retorna de Cerruto, también vivirá el engaño de la mujer muerta y de la casa abandonada. También serán los vecinos quienes le anoticiarán el fin del delirio:

[...] la casa que sus sueños reconstruyeron se derrumbó.

Se vio una estela de polvo en el lugar. Algún vecino forzó la puerta, había que actuar rápido, no era la primera vez que sucedía. Todos sabían que un anciano extraño se había adueñado de la casa abandonada diciendo que era suya y se lo habían permitido porque doña Eduviges Maygua, una anciana que estaba a punto de cumplir ciento dos años, había dicho que ese anciano extraño y silencioso no mentía. Habían tratado de advertirle del peligro, pero a él no le había importado. (2011: 171-172)

El final de la novela de Urquiola restaura el orden perdido, la recuperación de una paz que estuvo perturbada por la lluvia, esa lluvia que no paraba en la zona de Santa Fe al sur de la ciudad de La Paz, donde se ubicaba la casa de Esteban. La muerte, como un halo envolvente, cubre al personaje y, con el cese de la lluvia, el viejo encuentra la paz. 


\section{Dibliografía}

» Antezana, L. H. (1993). “Panorama de la narrativa y poesía bolivianas”. Revista Iberoamericana, vol. LIX, n. ${ }^{\circ}$ 164-165, pp. 577-591. Pittsburgh, Universidad de Pittsburgh.

"Antezana, L. H. (2000). “Cerruto en (el) “Cerco de penumbras”". En Cerruto, O., Cerco de penumbras. La Paz: Plural.

»Arguedas, A. (s/f). Pueblo enfermo. La Paz: Puerta del Sol.

»Ayllón, V. (2007). “Aruskipasipxañanakasakipunirakispawa: notas sobre dos poetas indígenas bolivianos contemporáneos". Revista Nuestra América, n.ํㅜ pp. 1-12. Porto: Universidade Fernando Pessoa.

"Cabrera, D. H. (2008). “Imaginarios de lo imaginario”. En Cabrera, D. H. (Coord.), Fragmentos del caos. Filosofía, sujeto y sociedad en Cornelius Castoriadis. Buenos Aires: Biblos.

"Calomarde, N. (2017). “Ficciones territoriales. Formas de un atlas latinoamericano". Revista RECIAL, año VIII, n. ${ }^{\circ} 12$, pp. 154-181. Córdoba, Centro de Investigaciones de la Facultad de Filosofía y Humanidades.

»Cerruto, O. (2000). “El círculo”. En Cerco de penumbras. La Paz: Plural.

»Cerruto, O. (2007). “Poema del mar imaginario”. En Obra poética. La Paz: Plural.

»Di Nucci, S.; Recoaro, N.; Grieco y Bavio, A. (2014). “Introducción”. En De la tricolor a la wiphala. Buenos Aires: Santiago Arcos.

" González Almada, M. (2016). “Territorialidades, textualidades. Torsiones y configuraciones en textos de Juan Pablo Piñeiro, Sebastián Antezana y Liliana Colanzi”. Revista Saga, n. ${ }^{\circ}$ 6, pp. 1-27. Rosario, Facultad de Humanidades y Artes.

» González Almada, M. (2017). Relaciones de poder, imaginarios sociales y prácticas identitarias en la narrativa boliviana contemporánea (2000-2010). Córdoba: Universidad Nacional de Córdoba.

"González Almada, M. (2017b). “Abigarramiento lingüístico, resistencia y traducción: la poesía de Mauro Alwa en el contexto de la literatura boliviana contemporánea". Mitologías hoy. Revista de pensamiento, crítica y estudios literarios latinoamericanos, vol. 16, pp. 1-16. Barcelona, Universidad Autónoma de Barcelona.

» Guattari, F. y Rolnik, S. (2013). Micropolítica. Cartografías del deseo. Buenos Aires: Tinta Limón.

»Ighina, D. (2005). “Nación, territorio, y construcción de identidades: el relato de la nacionalidad argentina de Ricardo Rojas”. Revista Diálogos, vol. 9, n. ${ }^{\circ} 3$, pp. 11-21. Maringá, DHI/PPH/UEM.

"Prudén, H. (2018). “Apuntes para una historia del mestizaje en Santa Cruz de la Sierra, Bolivia”. Revista Iberoamericana, año XVIII, n.․ 67, Berlín, disponible en https://journals.iai.spk-berlin.de/index.php/iberoamericana/article/ view/2446/2020

»Urquiola Flores, R. (2011). Lluvia de piedra. La Paz: Alfaguara.

»Urquiola Flores, R. (2016). “Escribir para leer”. En Zelaya Sánchez, Martín (Comp.), Flujo continuo. Primero leo, luego escribo. Las lecturas en la formación y 
vocación. La Paz: 3600.

»Saraceni, G. (2008). Escribir hacia atrás. Herencia, lengua, memoria. Rosario: Beatriz Viterbo.

»Wiethüchter, B. (1986). “Propuestas para un diálogo sobre el espacio literario boliviano". Revista Iberoamericana, vol. LII, n. ${ }^{\circ}$ 134, pp. 165-18o. Pittsburgh, Universidad de Pittsburgh.

»Williams, R. (2009). Marxismo y literatura. Buenos Aires: Las Cuarenta. 